\title{
Female Sexual Health: Barriers to Optimal Outcomes and a Roadmap for Improved Patient-Clinician Communications
}

\author{
Sheryl A. Kingsberg, PhD, Jonathan Schaffir, MD, ${ }^{2}$ Brooke M. Faught, DNP, WHNP-BC, IF, ${ }^{3}$ \\ JoAnn V. Pinkerton, MD, FACOG, NCMP, ${ }^{4}$ Sharon J. Parish, MD, ${ }^{5}$ Cheryl B. Iglesia, MD, FACOG, ${ }^{6}$ \\ Jennifer Gudeman, PharmD, Julie Krop, MD, ${ }^{7}$ and James A. Simon, MD ${ }^{8}$
}

\begin{abstract}
Background: Although sexual health can be considered a vital sign for overall health, several barriers prevent women from receiving proper medical counseling, support, and/or care for their sexual health needs and concerns. Methods: Experts in sexual health compiled research and experience on the impediments to women receiving adequate assessment and treatment for their sexual health. Specific solutions and a roadmap for overcoming such barriers and improving patient-clinician communication are presented.

Results: Social stigma around female sexuality remains in Western culture and as a result, women often avoid and/or are embarrassed to discuss their sexual health with their health care professionals (HCPs). Moreover, midlife women are typically unaware or have misconceptions about conditions that may adversely impact their sexual life, such as genitourinary syndrome of menopause and hypoactive sexual desire disorder. Without understanding there may be underlying medical conditions, there is also a lack of awareness that safe and effective treatments are available. Lack of training, tools, time, and limited treatment options impede HCPs from providing women with necessary sexual health support. Educating women, training HCPs, and providing communication tools to HCPs can facilitate effective dialog between patients and HCPs. More specifically, HCPs can be trained to initiate and maintain a sexual health conversation in a manner that is comfortable for women to convey sexual health needs and concerns, and for HCPs to correctly identify, diagnose, and treat the sexual problems of their female patients.
\end{abstract}

Conclusions: Solutions exist to address the barriers currently impeding patient-clinician interactions around sexual health.

Keywords: communication, education, menopause, sexual health, women

\section{Introduction}

$\mathbf{T}$ HE CONCEPT OF SEXUAL HEALTH has evolved significantly since the definition offered by the World Health Organization in $1975 .^{1}$ Although different definitions of the term continue to exist today, the general principles of "autonomy and pleasure and lack of coercion and lack of violence and a positive contribution to one's overall well-being," tend to thread across most definitions, offering useful guideposts for clinicians and researchers alike. ${ }^{2,3}$ Specifically, the assertion

\footnotetext{
${ }^{1}$ MacDonald Women's Hospital, University Hospitals Cleveland Medical Center, Cleveland, Ohio.

${ }^{2}$ Ohio State University College of Medicine, Columbus, Ohio.

${ }^{3}$ Women's Institute for Sexual Health, Nashville, Tennessee.

${ }^{4}$ University of Virginia Health System, Charlottesville, Virginia.

${ }^{5}$ Weill Cornell Medical College, White Plains, New York.

${ }^{6}$ MedStar Washington Hospital Center, Washington, District of Columbia.

${ }^{7}$ AMAG Pharmaceuticals, Waltham, Massachusetts.

${ }^{8}$ George Washington University, School of Medicine, IntimMedicine Specialists, Washington, District of Columbia.
}

(C) Sheryl A. Kingsberg et al. 2019; Published by Mary Ann Liebert, Inc. This Open Access article is distributed under the terms of the Creative Commons License (http://creativecommons.org/licenses/by/4.0), which permits unrestricted use, distribution, and reproduction in any medium, provided the original work is properly cited. 
that sexual health is a vital sign for overall health is a foundational principle guiding the issues raised in this article. Although "sexual health" may include issues related to having sex, such as contraception and sexually transmitted infections, this article will use the term specifically to refer to healthy female sexual function. Despite the previous assertion, many adult women in the United States today continue to go without the necessary medical counseling, support, and/or care to fully address their sexual health needs and concerns. This is especially true for women experiencing symptoms such as painful sex, decreased desire or pleasure, and/or orgasmic disorders.

Although adult women of all ages can experience distressing sexual health-related conditions or concerns, women through the menopausal transition and beyond tend to experience these conditions with greater frequency. ${ }^{4,5}$ Genitourinary syndrome of menopause (GSM), which includes vulvar and vaginal atrophy, ${ }^{6}$ is a highly prevalent medical condition associated with menopause ${ }^{7-9}$; typical symptoms include sexual pain, genital dryness, vaginal irritation/itch, and/or postcoital bleeding. These symptoms are often chronic, persisting through menopause, ${ }^{10-12}$ and have the potential to interfere with a woman's sexual activity, intimate relationships, lifestyle, and self-esteem. ${ }^{7,8,13-15}$ In fact, symptoms of GSM have been found to impact women's quality of life to a similar extent as arthritis, chronic obstructive pulmonary disease, asthma, or irritable bowel syndrome. ${ }^{16}$

Female sexual dysfunction (FSD), which refers to a number of sexual dysfunctions (e.g., loss of desire, decreased arousal, inability to reach orgasm and pain with sexual activity) that commonly overlap (Fig. 1), may be exacerbated by changes that occur around the menopausal transition for women. A hallmark criteria for all FSDs is personal distress, ${ }^{17}$ resulting in a reduced quality of life for affected women. For example, women with hypoactive sexual desire disorder (HSDD), the most common FSD, report low scores on medically recognized instruments that measure quality of life (including the SF-36 and SF-12 Health Surveys and the EQ-5D) ${ }^{18,19}$ that are comparable in magnitude to people suffering from back pain or diabetes. ${ }^{18}$

Gaps in care for these types of sexual health concerns occur for multiple reasons. For patients, reasons include so-

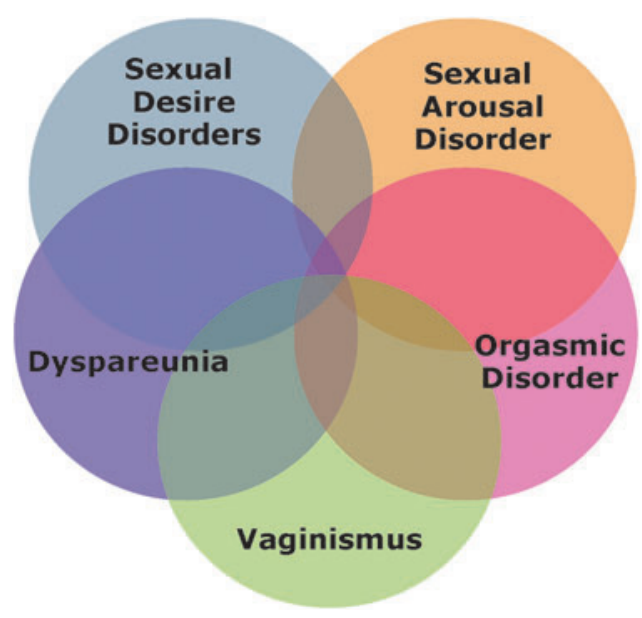

FIG. 1. Overlap of female sexual disorders. cial stigma and fear of broaching the topic, low awareness of the conditions and/or availability of effective treatments, and misperceptions about known treatments. For health care professionals (HCPs), suboptimal care may be because of time constraints; lack of training about diagnostic tools and treatment options; and costs, coverage, and regulatory/policy issues. Collectively, these issues often lead to inadequate sexual health outcomes for women.

The purpose of this article was to explore key obstacles to better outcomes in women's sexual health and suggest a roadmap for developing solutions to these barriers. Figure 2 outlines major barriers and the possible solutions that will be discussed in this article. Although we recognize that systemic cultural and regulatory/policy changes may be necessary to address certain challenges in the long term, the recommendations and considerations in this article focus on tangible actions HCPs can take to have a meaningful impact in the near term.

\section{Factors Contributing to Suboptimal Sexual Health Outcomes in Adult Women}

\section{Social stigma and conversation avoidance}

Based on cultural norms and biases, conversations about sex are sometimes thought of as taboo in American society and in many other cultures worldwide. This is especially true for women, and particularly when sex is for pleasure rather than reproductive purposes. Failure to have informative discussions about sex often leads to misperceptions about sex and sexuality, including a sense that pain or lack of interest in sexual activity is inevitable and nonmodifiable, which can also lead to women not seeking the care they need. In addition, women sometimes assume that older people do not, or should not, engage in sexual activity.

Despite society not fully appreciating the importance of sexuality in older individuals, studies indicate that sexuality and/or sexual activity is important to women at all stages of adulthood, including in the postmenopausal years. The Survey of Midlife Development in the United States found that $\sim 60 \%$ of women older than 60 years of age were sexually active, ${ }^{20}$ and another survey found that $22 \%$ of married women between 70 and 79 years old are sexually active. $^{21}$ In the AARP Survey of Midlife and Older Adults, $\sim 60 \%$ indicated that sex is a critical component to a good relationship ${ }^{22}$; and in another survey, sexual activity was positively associated with quality of life and successful aging. ${ }^{23}$ When assessing sexual activity in older adults, it is important to consider that sexual expression may extend beyond penile/vaginal intercourse. For many older individuals, sexuality is still important despite age-related difficulties such as erectile dysfunction (ED) or limited mobility, and for such couples, sexual activity may include other forms of intimacy or masturbation.

Female patients often apprehensive to discuss sex and sexual health with HCPs. Several U.S. and international surveys of women recently found that the majority of women surveyed did not discuss their sexual health-related symptoms with their HCPs, and discomfort and/or embarrassment with having this discussion was often cited as a reason for avoiding the conversation. ${ }^{7,24-26}$ This finding was consistent for women across different demographics, 
Patient barriers

\begin{tabular}{|l|}
\hline 1) Social stigma, media, \\
and misperceptions
\end{tabular}

Provider barriers

\section{Potential solutions}

- Educate women that FSDs and VVA/GSM are real medical conditions, not just inevitable aspects of aging

- Teach women about the range of treatments for FSDs and VVA/GSM

- Provide educational materials at initial and follow-up conversations on sexual and vaginal health

- Empower women with conversation tools to bring up sexual health concerns
FIG. 2. Patient-related and health care provider-related barriers aligned with potential solutions.

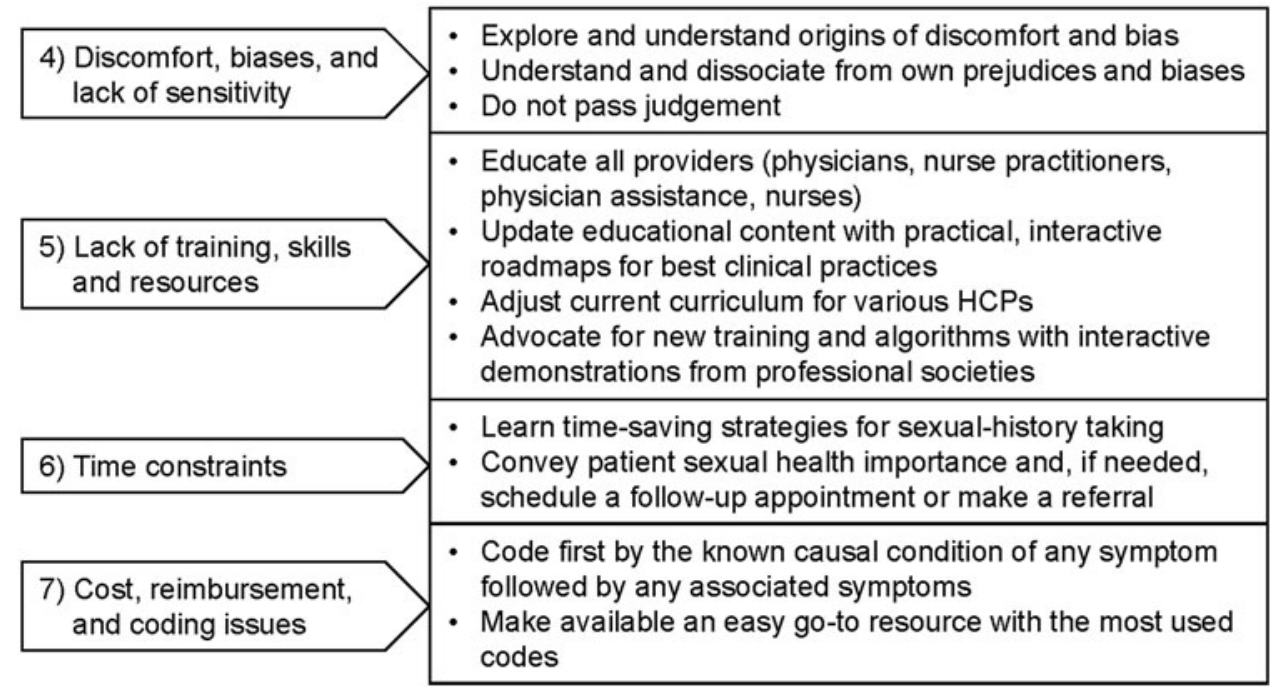

including age, sexual orientation, race/ethnicity, educational level, and relationship status. ${ }^{27}$

Previous negative experiences discussing sexual health with a clinician or concerns about how an HCP may react to the conversation often deter women from raising sexual health topics. Many fear that their concerns will be dismissed or considered unimportant. Furthermore, if they do raise a sexual health issue and feel it was not adequately addressed by the practitioner, women may perceive that their practitioner does not value their sexual health as a priority and/or believe that it is not amenable to treatment. $^{24,28,29}$

Women in internationally conducted focus groups reported that when they broached the subject of sexual health symptoms, their HCPs tended to display a lack of sensitivity to these symptoms affecting their quality of life. ${ }^{30}$ In one survey, $59 \%$ of women believed that other people do not want to hear about their "vaginal problems", ; and in another, more than one-third of women who sought help for sexual health issues felt their concerns were not adequately addressed or not taken seriously enough. ${ }^{25}$

Women may also wrongfully fear they are alone in experiencing these kinds of symptoms or that their HCPs will view them (and their sexual health concerns) as unimportant. Women may also be concerned that there is not enough time during their appointment to address such a sensitive and multifaceted topic.

Although some women would like to discuss their sexual health with their HCP, they may not know how to bring up the subject or may be unsure of which clinician they should discuss it with. No one specialty "owns" sexual health, which can lead to patient confusion about where to best seek help. Women may also be unsure of how to articulate their symptoms and may downplay the extent of their symptoms if they are uncomfortable discussing them.

Numerous surveys highlight that women tend to expect their HCPs to initiate dialog around sexual health. In the REal Women's VIews of Treatment Options for Menopausal Vaginal ChangEs (REVIVE) survey, $40 \%$ of women expected HCPs to initiate a conversation about their symptoms ${ }^{26}$; and in another recent survey, two-thirds of menopausal women agreed that HCPs should inquire regularly about sexual health. ${ }^{27}$

HCPs may forego initiating conversation on sexual health. Some HCPs do not initiate the conversation on sexual health because of a lack of confidence, personal discomfort, or a sense of discomfort on the part of the patient. ${ }^{29,30}$ Time constraints for office visits may also play a role in HCP hesitation to raise sexual health concerns. In addition, HCPs may take an inconsistent and/or avoidant approach to address the sexual health concerns of their older female patients, which may be because of an assumption that women's sexual function is less relevant beyond reproductive years. $^{28,31}$

In one survey, $73 \%$ of women said their primary care providers asked about sexual health only a few times or almost never. ${ }^{27}$ In the REVIVE survey, only $19 \%$ of menopausal women were asked by their HCPs about sexual health. ${ }^{26}$ In the Vaginal Health: Insights, Views and Attitudes 
(VIVA) survey, $50 \%$ of women stated their HCPs had not raised the topic of menopausal vaginal health. ${ }^{8}$

HCPs have self-reported on the frequency with which they discuss sexual health with patients. In a survey of OB/GYNs (obstetricians-gynecologists), two-thirds routinely asked about sexual activity, but only $40 \%$ asked about sexual concerns and $29 \%$ about sexual satisfaction. ${ }^{32}$ In a survey of nurses specifically caring for heart failure patients, $75 \%$ felt they had a responsibility to discuss sexual health with their patients. However, only $38 \%$ did so occasionally, $53 \%$ rarely, and $8 \%$ never assessed their patients' sexual health. ${ }^{33}$ Nurses cited a lack of knowledge regarding how to start the conversation as a reason for not discussing sexuality with patients. ${ }^{33}$

\section{Low awareness of sexual health conditions and/or availability of effective treatments}

Women often do not think of their sexual health concerns as a medical condition, which may be a reason why they do not raise the subject with their HCPs. In particular, many women do not recognize GSM as a common consequence of menopause, especially because symptoms, including vaginal dryness and dyspareunia, often do not present until several years after they stopped experiencing menstrual cycles. Instead, many women view GSM symptoms as an inevitable part of the aging process, rather than a medical condition amenable to treatment. Many women remain unaware that GSM symptoms, including dyspareunia, may be because of menopause, $, 8,14,24,26,34$ and the associated decreased levels of estrogens and other sex steroids. Similarly, surveys of participants in an HSDD registry found the most frequent reason women suffering from HSDD did not seek medical help was because they believed the symptoms were just a natural part of aging or being in a long-term relationship. $^{35}$

Lack of women's awareness of the availability of effective treatments for sexual health conditions also creates barriers. Despite the decades-long availability of effective vaginal estrogen therapies to treat GSM, the Women's EMPOWER survey found approximately two-thirds of participants were only somewhat familiar to unaware of effective treatments for their symptoms. ${ }^{24}$ Forty percent of participants in the aforementioned HSDD registry for women also reported delaying or foregoing professional help for their symptoms because they did not believe a treatment existed. $^{35}$

As with patients, many HCPs also remain unaware of available and approved treatment options; and some HCPs may even believe that certain sexual health conditions are not real medical conditions, are not within their purview, or are exaggerated by pharmaceutical companies.

\section{Treatment misperceptions among patients}

When women are aware of the availability of vaginal hormone therapy, including estrogens and prasterone (a synthetic form of dehydroepiandrosterone or DHEA), for treatment of GSM, they frequently have concerns about safety. The Women's Health Initiative (WHI), released in 2002, ${ }^{36}$ dramatically changed $\mathrm{HCP}$ and patient perceptions around the safety of estrogen use in menopause. The WHI, which studied oral conjugated estrogens (alone in hysterectomized women or with medroxyprogesterone acetate in nonhysterectomized women), has been widely criticized, most notably for the average age of enrollment being $>10$ years postmenopause. ${ }^{36}$ Since the early results from the WHI evaluating the oral standard dose of conjugated estrogens with medroxyprogesterone acetate, the "class labeling" for all estrogen-containing medications, including low-dose vaginal estrogens, has been required by the Food and Drug Administration (FDA). The North American Menopause Society (NAMS) and others have advocated for modification of labeling for low-dose vaginal estrogens based upon the minimal systemic exposure with blood levels remaining within the normal postmenopausal range and without increased risk of heart disease, stroke, blood clots, or probable dementia. ${ }^{37}$ However, apprehension about estrogen therapy because of fear and the unchanged boxed warning remains for many women. As a result, HCPs may find it challenging to overcome this negative perception when counseling their patients on low-dose vaginal treatment options.

Women may also receive inaccurate information about the efficacy of treatments marketed to address sexual health conditions. Unfortunately, the popularization of claims about unproven treatments is prevalent, as are negative perceptions about effective treatments. Celebrities and others in the public spotlight may confuse women by touting the supposed benefits of unproven methods, such as "vaginal rejuvenation," while dismissing well-studied, effective therapies with longer term safety data. Of importance, the FDA recently warned not to use energy-based devices (radiofrequency or laser) that have received clearance for general gynecologic indications for vaginal "rejuvenation," cosmetic vaginal procedures, or nonsurgical vaginal procedures to treat symptoms related to menopause, urinary incontinence, or sexual function until further clinical trial data confirms efficacy and safety for specific issues. ${ }^{38}$ In addition, alternative therapies, such as "bioidentical" compounded hormones and plateletrich plasma are promoted as "natural" treatments for GSM, but are not regulated by FDA with regard to efficacy, safety, or marketing claims, in marked contrast to FDA-approved treatment options.

\section{Lack of HCP training, tools, and treatment options}

Most HCPs, including physicians, physician assistants, nurse practitioners, and nurses, receive limited formal sexual health training. Although sexual medicine has grown substantially in the past 20 years, aspects of training continue to lag behind scientific and clinical knowledge in the field. ${ }^{39}$ In addition, the time devoted in medical school to sexual health and function and all of its complexities has dramatically decreased in medical schools. ${ }^{40}$ A 2016 review of existing and future educational needs in graduate and postgraduate medicine found that sexual medicine objectives were included in only a few residency programs. ${ }^{39}$ Even when these requirements were included, they were typically defined in a very general fashion (e.g., family medicine guidelines call for "teaching of human sexuality"; gynecology residents receive training in contraception, infertility, menopause, high-risk sexual behavior, and specific sexual medicine skills such as sexual history taking). ${ }^{39}$ Of note, even training for urogynecologists focuses only on pelvic floor dysfunction. Professional societies may offer optional formal training on FSDs; 
in addition, continuing medical education (CME) may be sought by HCPs to address gaps in knowledge. ${ }^{39}$ However, some HCPs may not be motivated to seek out additional "optional" CME training, for which availability also varies.

Despite the fact that medical society guidelines and associated tools exist for screening common sexual health conditions like HSDD, HCPs may be unfamiliar with them or may fail to use them for patient diagnosis. HCPs may also not be familiar with educational and/or referral resources for their patients, which may prevent them from addressing sexual health at an office visit. Office-visit time constraints may contribute to HCP hesitating to raise sexual health concerns, especially if they have not received training and tools to help conduct these conversations in an efficient manner.

In addition, clinicians may feel they have limited therapeutic options to use to treat certain sexual health conditions. Kingsberg et al. recently provided an extensive overview of the psychosocial and pharmacologic (hormonal and nonhormonal) treatments for FSD, including those for HSDD, female sexual arousal disorder, and female orgasmic disorders, and found treatments in this area are severely lacking. ${ }^{41}$ There is currently one FDA-approved treatment for HSDD (flibanserin) and no approved treatments for arousal or orgasmic disorders. Although the indicated population for flibanserin in HSDD is currently limited to premenopausal women only, data showed that flibanserin improved sexual function in postmenopausal women with HSDD. ${ }^{42,43}$ In addition, a variety of over-the-counter lubricants and moisturizers, vaginal estrogens, and two nonestrogen therapies (oral ospemifene or vaginal prasterone) are available for GSM and/or dyspareunia, although surveys indicate that all GSM treatments are underutilized. ${ }^{24,26}$

Considerable public debate has ensued over a perceived gender disparity in sexual health drug development, as there have been approved treatments for men with ED for 20 years. This may be because of differences in the underlying etiologies of these conditions, the additional regulatory burden of evaluating separately pre- and postmenopausal women, or some combination thereof. Regardless, the advent of safe, effective treatment options for men suffering from ED, coupled with frequent direct-to-consumer advertising, has largely moved a previously stigmatized condition into open and acceptable dialog, with men now comfortable vocalizing their sexual health needs to their HCPs and HCPs feeling equipped with therapeutic options to address the concern. A national probability sample of 1550 women and 1455 men aged 57-85 years revealed that sexual dysfunction has negative concomitant outcomes and that it is more common in women $(11.3 \%-43.3 \%)$ than men $(2.9 \%-36.9 \%) .{ }^{44}$ In addition, the Fourth International Consultation on Sexual Medicine (2015) found that women were more likely to have multiple sexual dysfunctions than men. ${ }^{45}$ Given the nearly two decades that male sexual health has been a topic of frequent and comfortable public discourse, and the recognition that FSD is more prevalent, we assert that female sexual health must be similarly recognized, valued, and prioritized as a component of overall health.

\section{Cost, coverage, and regulatory/policy issues}

Cost is a consideration for any treatment, particularly if managed care organizations and other gatekeepers of health care dollars deprioritize the relative importance of a therapeutic area, as is often the case with female sexual health. Clinicians may be reluctant to prescribe effective treatments if they suspect the cost to the patient at the pharmacy may be higher than she is willing or able to pay. As a result, they may recommend less expensive (although ineffective) alternatives, turn to unregulated compounding, or recommend over-the-counter vaginal lubricants and moisturizers (sometimes less expensive), which are effective for mild symptoms of vaginal dryness and/or dyspareunia, but do not treat the underlying vulvovaginal changes associated with decreased sex steroids that lead to dyspareunia. Moreover, given their patients' numerous and varied insurance benefits, HCPs may find it difficult to know offhand which treatments are covered by which insurance plans. Cost may also be a barrier for referrals to other clinicians for counseling and cognitive-behavior therapy for treating sexual dysfunction.

Relatedly, reimbursement of treatments for women's sexual health-related conditions is often lacking. Medicare had considered dyspareunia as a result of menopause as a sexual dysfunction and did not provide coverage for medical treatments, as has occurred with other "lifestyle" conditions. NAMS, International Society for the Study of Women's Sexual Health (ISSWSH), and the American College of Obstetricians and Gynecologists (ACOG) had advocated for its coverage. In May 2018, the Centers for Medicare and Medicaid sent clarification to NAMS, ISSWSH, ACOG, and others committed to women's health that drugs approved by the FDA for the treatment of moderate to severe dyspareunia, a symptom of vulvar and vaginal atrophy because of menopause, are not excluded from Medicare Part D under $\S 1860 D-2(e)(2)(A)$ of the Social Security Act. $^{46}$

The necessity of a boxed warning for safety, currently required as part of the label for low-dose, vaginal estrogencontaining therapies to treat GSM, is a further obstacle that has been called into question by gynecologic HCPs in recent years. $^{37}$ An oral selective estrogen receptor modulator, ospemifene was FDA approved in 2013 with a modified boxed warning; an intravaginal medication containing prasterone was FDA approved in 2016 without a boxed warning that may facilitate acceptance by patients. However, boxed warnings remain for all vaginal estrogen therapies.

Although no significant alcohol interaction with flibanserin was reported in the large pivotal efficacy and safety trials, ${ }^{42,43}$ in a small alcohol challenge study, 4 of 23 men $(17 \%)$ experienced hypotension or syncope requiring therapeutic intervention. ${ }^{47}$ As a result, a Risk Evaluation and Mitigation Strategy (REMS) program for flibanserin was established. This REMS requires prescribers to become certified to prescribe (and pharmacists to dispense), which has limited access for women. In addition, the recent approval of flibanserin in Canada does not have an alcohol intake restriction as it does in the United States. At present, there is only one other product in development (bremelanotide) for treating HSDD that has been submitted to the FDA with a Prescription Drug Fee User Date of June 2019. The FDA should maintain warnings and restrictions on such medications that are based on objective criteria and not hold medications for sexual dysfunction to a different standard. 


\section{Overcoming Barriers: Practical Recommendations to Encourage Better Clinical Interactions}

As outlined previously, barriers to optimal sexual health outcomes for women are numerous, complex, and often interrelated. We recognize some problems are more solvable than others. Although we support efforts to address all obstacles, a necessary and achievable first step is to foster more open and informed dialog about sexual health between women and their HCPs. This simple but critical measure will improve women's health outcomes ${ }^{48,49}$ and can be accomplished in the near future. Therefore, the specific recommendations of this article address strategies to enable better $\mathrm{HCP}$-patient communication with a focus on skills training for professionals and patient education and empowerment programs (Fig. 2). We believe that several barriers need to be addressed with such training and education.

\section{Training HCPs and facilitating communication}

All HCPs (physicians, nurse practitioners, physician assistants, mental health professionals, nurses, etc.) who provide health care for women can benefit from training in the basics of female sexual health and dysfunctions in combination with communication skills training to facilitate successful, candid discussion about sexuality. More specifically, HCP training should focus on integrating knowledge about sexual health with skills for counseling patients and shared decision-making based on individual needs and goals.

HCP training. Knowledge acquisition about basic sexual health content and fundamental interviewing/communication skills should begin at the earliest stage of professional training, for example, in medical and professional schools. Despite the ever-growing competition for time with exponential growth in knowledge to be learned, advocacy efforts must be expanded to protect sexual health content and communication skills in core curricula. Further training (or in some cases, even initial training) can occur in residency or other postgraduate training. HCPs already in clinical practice who need basic training or are interested in enhanced training in female sexual medicine can seek out CME programs on sexual health education. Women's health and/or sexuality- related professional associations can also play an important role in creating and disseminating resources and best practices for practitioners (Table 1) on how to counsel patients on sexual health in the clinical setting. ${ }^{39}$

Such best practices can be accomplished in almost any office visit setting. HCPs can first legitimize the importance of assessing sexual function and normalize the discussion by including it as part of the routine medical history. One suggestion to put patients at ease may be to mention at the outset that many patients have sexual health concerns or symptoms, providing an opening for them to ask if the patient has similar concerns. HCPs can use open-ended questions about sexual concerns instead of "yes/no" questions, which tend to hinder women's ability to accurately describe symptoms or concerns. In addition, open-ended inquiries give patients permission to talk about their sexual concerns. Routine discussion of sexual health allows HCPs to reassure women that some feelings and symptoms are common and legitimate. Any problems related to sexual response, including desire, arousal, orgasm, and pain can be explored, along with potential treatment options. This is an efficient model for HCPs to simultaneously educate women about normal sexual response and assess for problems with desire, arousal, orgasm, or pain.

The PLISSIT model is a helpful tool for discussing sexual health or concerns with patients. ${ }^{50,51}$ PLISSIT stands for permission $(\mathrm{P})$, limited information (LI), specific suggestions (SS), and intensive therapy (IT), as outlined in Table 2. When conducting an interview, the Partnership (P), Empathy (E), Apology (A), Respect/Reflect/Reinforce (R), Legitimize (L), Support (S) (PEARLS) model can also provide a useful structure to facilitate open communication (Table 2). ${ }^{52}$

Essential clinical competencies for communication about sexual health concerns include the ability to initiate a direct and concise conversation about sexual health in a space that ensures privacy and comfort. For example, the HCP and patient should both be seated face-to-face with the patient clothed. The HCP should complete a brief sexual health history, discuss any concerns, and close the conversation with shared decision-making and a suggestion for a follow-up appointment to further assess and treat. Alternatively, the $\mathrm{HCP}$ can provide a referral to a sexual medicine expert or sex

Table 1. Relevant Health Care Professional Resources

Association Title

American College of Obstetricians and Gynecologists 54

American College of Obstetricians and Gynecologists ${ }^{17}$

International Society for the Study of Women's Sexual Health ${ }^{59}$

International Society for the Study of Women's Sexual Health ${ }^{60}$

International Consultation of Sexual Medicine $^{61}$

International Consultation of Sexual Medicine ${ }^{62,63}$

North American Menopause Society ${ }^{64}$

North American Menopause Society ${ }^{65}$
Committee Opinion 706: Sexual Health

Practice Bulletin 119: Female Sexual Dysfunction

Process of Care for Management of Hypoactive Sexual Desire Disorder in Women

Hypoactive Sexual Desire Disorder: International Society for the Study of Women's Sexual Health Expert Consensus Panel Review

Definitions of Sexual Dysfunctions in Women and Men: A Consensus Statement from the Fourth International Consultation on Sexual Medicine 2015

Toward a More Evidence-Based Nosology and Nomenclature for Female Sexual Dysfunctions: Parts I and II

Management of symptomatic vulvovaginal atrophy: 2013 position statement of The North American Menopause Society Sexual Health website for menopausal women 
Table 2. Models for Open Clinician-Patient Communication about Sexual Health

\begin{tabular}{|c|c|c|c|}
\hline Model & Abbreviation & Meaning & Example \\
\hline \multirow[t]{4}{*}{ PLISSIT $^{50,51}$} & $\mathrm{P}$ & $\begin{array}{l}\text { Permission for the patient to discuss their sexual } \\
\text { concerns (confirming normalcy), permission } \\
\text { to continue doing what they are doing, OR } \\
\text { permission to begin sexual assessment with } \\
\text { open-ended questions }\end{array}$ & $\begin{array}{l}\text { "Many of my patients have problems with } \\
\text { their sexual health, what concerns you } \\
\text { about your sexual health?" }\end{array}$ \\
\hline & LI & $\begin{array}{l}\text { Limited information provided about } \\
\text { physiological changes }\end{array}$ & $\begin{array}{l}\text { Provide information on the normal and } \\
\text { pathologic changes that may affect } \\
\text { sexuality }\end{array}$ \\
\hline & SS & $\begin{array}{l}\text { Specific suggestions for care plan based on } \\
\text { open-ended questions }\end{array}$ & $\begin{array}{l}\text { "Use of lubricant during intercourse is a } \\
\text { helpful way to reduce pain from } \\
\text { penetration that is a result of dryness" }\end{array}$ \\
\hline & IT & Intensive therapy for sexual problems, if needed & $\begin{array}{l}\text { Psychotherapy may be needed for a patient } \\
\text { who has been sexually abused }\end{array}$ \\
\hline \multirow[t]{6}{*}{ PEARLS $^{52}$} & $\mathrm{P}$ & Partnership (HCP and patient in this together) & $\begin{array}{l}\text { "Whatever sexual problems you are having, } \\
\text { we can work on them together" }\end{array}$ \\
\hline & $\mathrm{E}$ & Empathy (express understanding to the patient) & $\begin{array}{l}\text { "I understand that what you are going } \\
\text { through is very depressing", }\end{array}$ \\
\hline & A & Apology (acknowledge any wrongdoing) & $\begin{array}{l}\text { "I'm sorry you had to wait so long to see } \\
\text { me" }\end{array}$ \\
\hline & $\mathrm{R}$ & $\begin{array}{l}\text { Respect/Reflect/Reinforce (acknowledge the } \\
\text { patient's suffering, difficulties, etc.) }\end{array}$ & "That sounds like a very difficult situation" \\
\hline & $\mathrm{L}$ & $\begin{array}{l}\text { Legitimize (acknowledge the legitimacy of the } \\
\text { complaint) }\end{array}$ & $\begin{array}{l}\text { "What you complain of is a real medical } \\
\text { condition" }\end{array}$ \\
\hline & $\mathrm{S}$ & $\begin{array}{l}\text { Support (convey the HCP will not abandon the } \\
\text { patient) }\end{array}$ & $\begin{array}{l}\text { "I will do whatever I can to help you" } \\
\text { "These concerns are common and I have } \\
\text { personally helped many women address } \\
\text { them" }\end{array}$ \\
\hline
\end{tabular}

HCP, health care professional.

therapist who can address more complex concerns. The International Urogynecological Association (IUGA) and International Continence Society (ICS) stress that sexual concerns should be addressed routinely and in a recent report suggested an educational process similar to the above to be used in women with pelvic floor dysfunction, given that most pelvic floor dysfunctions are believed to negatively affect sexual health. ${ }^{53}$ The ACOG Committee Opinion on sexual health, meant to increase awareness of the importance of addressing women's sexual health in routine practice, provides a listing of questions to be utilized during sexual history taking. ${ }^{54}$ We advocate that all HCPs of any specialty should be able to initially address sexual health issues, or if not comfortable doing so, have a streamlined, care-path referral in place as part of their routine practice.

As noted in the PLISSIT model, beyond providing basic information and suggestions, many HCPS may still want to refer a patient to qualified sexuality specialists. Appropriate sexual health referrals could apply to HCPs in any specialty area. For example, a neurologist treating a patient with multiple sclerosis could discuss sexual health with her patients, and if lacking in expertise, be able to provide them with some appropriate HCP recommendations. Professional associations could help HCPs direct women to sexual health specialists by having a mechanism for HCPs to identify appropriate professionals by location and areas of expertise. ${ }^{55}$

Increasing HCPs' familiarity with appropriate International Classification of Diseases (ICD) codes for FSDs (Table 3) will help address the barrier to care caused by HCPs avoidance of assessing sexual concerns because of lack of awareness that there are corresponding billing codes they can easily use for ensuring insurance coverage and payment of visits and treatments for patients with sexual health-related concerns. FSDs listed in the Diagnostic and Statistical Manual of Mental Disorder, Fifth Edition (DSM-5) are included in Mental, Behavioral and Neurodevelopmental Disorders ICD-10 codes that begin with the letter "F." "F", codes are appropriate for mental and behavioral health care diagnoses. HCPs in the clinical setting should consider correlating symptom codes, which may be more appropriate when patients present with symptoms of FSD, before an actual diagnosis is made. Various other codes may also be considered to support the diagnostic workup of FSD and referral to pelvic floor physical therapy. The known causal condition of any symptom should be coded first, followed by any associated symptoms of the condition. For example, low libido because of pain with intercourse would be coded first by pain with intercourse and then for low libido (Table 3). HCPs should always check with a billing and coding specialist for the most recent information.

Establishing and maintaining patient trust. A theorybased qualitative study of women older than 50 years of age found that women who wanted to communicate with their HCPs about sexual health would only do so if they felt comfortable and trusted their provider. ${ }^{56}$ HCPs can build rapport with a patient by asking questions about sexual health as comfortably as they would ask other health questions, by not rushing them through the discussion, by remaining nonjudgmental, and by assuring them that what is discussed will remain confidential ${ }^{29,56}$ and that what they are experiencing is normal. Specifically, a matter-offact attitude, ${ }^{29}$ explanation that these are common conditions, 
Table 3. International Classification of Diseases, Tenth Revision (ICD-10)

and Current Procedural Terminology Codes ${ }^{\text {a }}$ for Female Sexual

and Pelvic Floor Dysfunctions and Genitourinary Syndrome of Menopause

Diagnosis or reason

Code

Female sexual dysfunction (DSM-IV diagnosis)

Hypoactive sexual desire disorder

F52.0

Sexual aversion disorder

F52.1

Female sexual arousal disorder

F52.22

Female orgasmic disorder

Dyspareunia not due to a substance or known physiological condition

F52.31

Vaginismus not due to a substance or known physiological condition

F52.6

Interest and arousal disorders

Low libido

F52.5

Vaginal dryness

R68.82

Vaginal dryness, menopausal

N89.8

Persistent genital arousal disorder

N95.1

N94.89

Pain disorders

Dyspareunia

N94.1

Vaginismus

N94.2

Vulvodynia (other, unspecified)

N94.818/819

Vulvar vestibulitis

Atrophic vaginitis/genitourinary syndrome of menopause

N94.810

N95.2

Pelvic/perineal pain

$\mathrm{R} 10.2$

Pain in joint, pelvic region, and thigh

M25.559

Additional codes that may support physical therapy

Weak pelvic floor muscles

N81.89

M62.50

M62.81

Generalized muscle weakness

N94.89

High tone pelvic floor dysfunction

M62.838

Spasm of muscle

M54.5

Unspecified disorder of muscle, ligament, or fascia

M62.9

Additional codes that may support bloodwork

Symptomatic menopausal states

N95.1

Premenstrual tension syndromes

N94.3

Fatigue

R53.83

Apathy

R45.3

Low testosterone in female

E34.9

Emotional lability

R45.86

Weight gain

R63.5

Common CPT codes in sexual medicine

Wet prep

87210

83986

Vaginal pH

99000

Trigger point injections

$<3$ muscles

20552

$3+$ muscles

20553

Colposcopy/vulvoscopy

Vulva

Vulva with biopsy

56821

Biopsy

Vulva/perineum

56605

Additional lesions of vulva/perineum

56606

Vagina

57100

Extensive biopsy of vagina requiring suturing

57105

Perineometry (biofeedback)

90911

Pelvic floor physical therapy assessment

Low complexity

97161

Moderate complexity

97162

High complexity

97163

Fractional $\mathrm{CO}_{2}$ laser therapy ${ }^{\mathrm{b}}$

58999

\footnotetext{
${ }^{a}$ Regarding coding: Every reasonable effort has been made to ensure the accuracy of the information within this article. However, the ultimate responsibility for compliance with Medicare rules and regulations lies within the provider of services.

${ }^{\mathrm{b}}$ Unlisted procedure female genital system (nonobstetric).

DSM-IV, Diagnostic and Statistical Manual of Mental Disorder, Fourth Edition.
} 
and reassurance that they are treatable may help women relax when discussing these topics.

HCPs may initially feel confused by the common overlap of sexual concerns (i.e., problems with desire, arousal, and orgasm [Fig. 1]). However, simple inquiry regarding which problem developed first or is most distressing typically informs treatment decisions. Once a sexual health concern has been identified and addressed, HCPs should continue to check in with patients and discuss issues of sexual health on subsequent visits. At any stage, conversations about sexual health may be enhanced by providing credible and accurate educational information and resources to women.

Guidance should be given to HCPs to begin conversations on sexual health without any assumptions about sexual activity, sexual orientation, relationship status, or any other topics that would impede a connection with the patient, ${ }^{48}$ or could make them feel judged or ashamed. It is also important for HCPs to be sensitive and not pass judgment on women who may be engaged in sexual activity with women, men, or both, or self stimulation, and not assume that they are in a monogamous heterosexual relationship. ${ }^{57}$

Developing and using language that puts women at ease is also helpful in building trust. For example, postmenopausal women do not consider "vaginal atrophy" a suitable term for vaginal discomfort, ${ }^{8,14}$ and they may be less likely to discuss it if this language is used. Using plain language, props, and/or illustrative aids, and describing sexual anatomy and physiology in simple terms may help to make the explanations clearer. For instance, clinicians may want to use the terms "low libido" or "low desire" rather than HSDD. Many HCPs also find it beneficial to provide patients with a hand mirror to show the external genital changes that have occurred, or advise that their vaginal $\mathrm{pH}$ is alkalotic instead of acidic, both of which may also help make the patient and HCP feel more comfortable.

In addition, HCPs should be encouraged to explore the origins of their discomfort, ${ }^{31}$ and to understand their own biases through unconscious bias and empathy training exercises. They should also be aware of how their own personal views about sexuality could impact their interaction with patients, so they can dissociate these personal biases and views from their discussions with patients. ${ }^{29}$ A small survey of women 40-75 years old found that women were more likely to discuss sexual health when the HCP did not make assumptions and appeared nonjudgmental. ${ }^{57}$

Overcoming time constraints. To overcome time constraint issues, HCPs can be trained on time-saving strategies that can be implemented at various stages of the initial office visit. A practical suggestion is to first take a sexual history using open-ended questions (as opposed to "yes/no" questions), which can convey a great deal of information in a relatively short amount of time ( $\leq 5$ minutes). In addition, HCPs can use preformulated questionnaires, such as the decreased sexual desire screener, meant to facilitate the diagnosis of HSDD when clinician-patient time is limited. ${ }^{58}$ Clinicians could consider questionnaires before seeing patients in the office to save time; however, follow-up on the questionnaire during in-office, clinician-patient interaction is critical, as not all patients are comfortable with providing written documentation on this sensitive subject. HCPs should also address the most important topics that can be covered in a limited amount of time. Instead of feeling pressured to cover it all in one visit, they should convey to patients that their sexual health is important and encourage a follow-up appointment that focuses solely on sexual health concerns. Providing written information for patients to review may help facilitate a patient's return visit.

Making the most of medical intake. In addition to sexual health being addressed in routine medical history, other strategies to assess sexual health concerns include adding prompts in the electronic medical record or including sexual health questions on medical intake forms. ${ }^{57}$ Although some women may be concerned that inclusion of such information in an electronic record violates their privacy, they should be reassured that such information is kept secure and confidential, and only accessible by their health care providers. A system similar to that in behavioral health would be useful in which only HCPs who will be involved with the sexual issue have access to that aspect of the health record. In addition, clinicians can ask specific questions about phases of the sexual response (desire, arousal, orgasm) and pain to provide examples of the level of detail that they would like the patient to address.

Professional societies could develop creative new tools for HCPs to use in practice such as (1) clinical practice guidelines for diagnosis, management, and treatment, (2) summaries of well-conducted clinical trials, (3) ice-breaker videos, or (4) phrases to help prompt clinicians to initiate conversations that work. Clinical study summaries for HCPs can quickly summarize the main points of studies, conveying the most important data supporting the management and treatment of pertinent sexual health issues. Ice breakers and conversation prompts can offer tangible examples to help clinicians successfully broach sexual health topics with their patients.

\section{Educating and empowering patients}

In addition to offering education to HCPs, women themselves should be better educated and empowered to discuss sexual health. Educational tools explaining normal anatomy, biological and etiological factors, and sexual response, as well as possible causes of dysfunction, should clear up misconceptions and emphasize that sexual health-related conditions are common, real, and sometimes, as in the case of GSM, chronic and progressive. Using a hand-held mirror as standard of care could allow women to see and be empowered about their own anatomy. Credible, easy-to-understand information should be easily available in person at the clinician's office and online. Having easily available educational materials in the office also demonstrates a HCP's comfort and awareness of sexual health treatments. In fact, a recent international survey found that nearly half of menopausal women with vaginal discomfort would have liked information or a pamphlet on the topic to help decide whether to consult with their physician about it. ${ }^{7}$

Most importantly, better information, resources, and tools can help women to believe that sexual health is a critical part of their overall health and well-being and should be regarded with importance similar to other aspects of health. When patients are empowered this way, they may feel more 
competent to manage their own health and may be more likely to follow through with treatment. ${ }^{55}$

\section{Concluding Remarks}

Sexual health is as important as any other facet of health and should receive the same level of attention. The opportunity for women to freely and fully discuss their sexual health needs, concerns, and potential treatment options with a trusted HCP is critical for improving sexual health outcomes. Despite the role of sexual health as a vital sign for overall health and quality of life, the topic is unfortunately too often left unaddressed by both HCPs and female patients. Effective therapy is available. Many cultural, educational, and practical barriers contribute to the substantial under-recognition and under-treatment of sexual health-related conditions affecting women. Enhanced HCP training and support for better-guided conversations with patients and greater patient education and confidence are important starting points for developing necessary solutions to ensure optimal sexual health outcomes in women.

\section{Author Disclosure Statement}

S.A.K. is a consultant for, a scientific advisory board member, or a clinical investigator for AMAG, Dare, Duchesnay, Emotional Brain, Endoceutics, GTx, IVIX, Lupin, Materna, Palatin Technologies, Pfizer, Sermonix, Strategic Scientific Solutions (SST), TherapeuticsMD, and Valeant. J.S. is a clinical investigator for Viveve. B.M.F. served as a consultant for AMAG, Lupin, and Duchesnay; has served on the speaker's bureau for AMAG, Duchesnay, and Valeant; and is currently a clinical investigator for Ipsen Innovations. J.V.P. has received research support (in the past 3 years; paid to the University of Virginia) from TherapeuticsMD. S.J.P. is a member of the advisory board for AMAG; consults for Dare, JDS Therapeutics, Sprout, Strategic Scientific Technologies (SST), TherapeuticsMD and Proctor \& Gamble; and has received writing support from AMAG and TherapeuticsMD. C.B.I. reports no disclosures. J.A.S. has served (within the last year) or is currently serving as a consultant/advisor to AbbVie, Allergan Plc, AMAG, Ascend Therapeutics, Azure Biotech, Millendo Therapeutics, Nuelle, Radius Health, Regeneron, Roivant Sciences, Sanofi SA, Sebela, Sermonix, Shionogi, Symbiotec Pharmalab, TherapeuticsMD, and Valeant; has received (within the past year or currently) grant/research support from AbbVie, Allergan Plc, Agile Therapeutics, Bayer Healthcare, New England Research Institute, ObsEva SA, Palatin Technologies, Symbio Research, and TherapeuticsMD; has also served (within the last year or currently) on the speaker's bureaus of Novo Nordisk, Shionogi, and Valeant; and is a stockholder (direct purchase) in Sermonix Pharmaceuticals. J.G. and J.K. are employees of AMAG Pharmaceuticals. AMAG Pharmaceuticals supported the medical writing assistance of Kathleen Ohleth, $\mathrm{PhD}$, CMPP of Precise Publications, LLC.

\section{References}

1. World Health Organization. Sexual health document series. Defining sexual health: Report of a technical consultation on sexual health 28-31 January 2002. Available at: www .who.int/reproductivehealth/topics/gender_rights/defining_ sexual_health.pdf Accessed February 23, 2018.
2. American Sexual Health Association. Understanding sexual health. Available at: www.ashasexualhealth.org/sexualhealth Accessed April 25, 2018.

3. American Association of Sexuality Educators Counselors $\&$ Therapists (AASECT). How we define sexual health. Available at: www.aasect.org/how-we-define-sexual-health Accessed April 25, 2018.

4. Shifren JL, Monz BU, Russo PA, Segreti A, Johannes CB. Sexual problems and distress in United States women: Prevalence and correlates. Obstet Gynecol 2008;112:970 978.

5. Lindau ST, Schumm LP, Laumann EO, Levinson W, O'Muircheartaigh CA, Waite LJ. A study of sexuality and health among older adults in the United States. N Engl J Med 2007;357:762-774.

6. Sarrel PM, Portman D, Lefebvre P, et al. Incremental direct and indirect costs of untreated vasomotor symptoms. Menopause 2015;22:260-266.

7. Nappi RE, Kokot-Kierepa M. Women's voices in the menopause: Results from an international survey on vaginal atrophy. Maturitas 2010;67:233-238.

8. Nappi RE, Kokot-Kierepa M. Vaginal health: Insights, views \& attitudes (VIVA)-Results from an international survey. Climacteric 2012;15:36-44.

9. Constantine GD, Graham S, Clerinx C, et al. Behaviours and attitudes influencing treatment decisions for menopausal symptoms in five European countries. Post Reprod Health 2016;22:112-122.

10. Huang AJ, Moore EE, Boyko EJ, et al. Vaginal symptoms in postmenopausal women: Self-reported severity, natural history, and risk factors. Menopause 2010;17:121-126.

11. Huang AJ, Grady D, Jacoby VL, Blackwell TL, Bauer DC, Sawaya GF. Persistent hot flushes in older postmenopausal women. Arch Intern Med 2008;168:840-846.

12. Barnabei VM, Grady D, Stovall DW, et al. Menopausal symptoms in older women and the effects of treatment with hormone therapy. Obstet Gynecol 2002;100:1209-1218.

13. Erekson EA, Li FY, Martin DK, Fried TR. Vulvovaginal symptoms prevalence in postmenopausal women and relationship to other menopausal symptoms and pelvic floor disorders. Menopause 2016;23:368-375.

14. Simon JA, Kokot-Kierepa M, Goldstein J, Nappi RE. Vaginal health in the United States: Results from the Vaginal Health: Insights, Views \& Attitudes survey. Menopause 2013;20:1043-1048.

15. Huang AJ, Luft J, Grady D, Kuppermann M. The day-today impact of urogenital aging: Perspectives from racially/ethnically diverse women. J Gen Intern Med 2010;25: 45-51.

16. DiBonaventura M, Luo X, Moffatt M, Bushmakin AG, Kumar M, Bobula J. The association between vulvovaginal atrophy symptoms and quality of life among postmenopausal women in the United States and Western Europe. J Womens Health (Larchmt) 2015;24:713-722.

17. American College of Obstetricians and Gynecologists. Female sexual dysfunction. Practice Bulletin No. 119. Obstet Gynecol 2011;117:996-1007.

18. Biddle AK, West SL, D'Aloisio AA, Wheeler SB, Borisov NN, Thorp J. Hypoactive sexual desire disorder in postmenopausal women: Quality of life and health burden. Value Health 2009;12:763-772.

19. Leiblum SR, Koochaki PE, Rodenberg CA, Barton IP, Rosen RC. Hypoactive sexual desire disorder in postmenopausal women: US results from the Women's International 
Study of Health and Sexuality (WISHeS). Menopause 2006; 13:46-56.

20. Thomas HN, Hess R, Thurston RC. Correlates of sexual activity and satisfaction in midlife and older women. Ann Fam Med 2015;13:336-342.

21. Schneidewind-Skibbe A, Hayes RD, Koochaki PE, Meyer J, Dennerstein L. The frequency of sexual intercourse reported by women: A review of community-based studies and factors limiting their conclusions. J Sex Med 2008;5: 301-335.

22. Fisher L, Anderson O, Chapagain M, Montenegro X, Smoot J, Takalkar A. Sex, romance, and relationships: 2009 AARP survey of midlife and older adults. 2010. Available at: https://assets.aarp.org/rgcenter/general/srr_09.pdf Accessed February 10, 2018.

23. Thompson WK, Charo L, Vahia IV, Depp C, Allison M, Jeste DV. Association between higher levels of sexual function, activity, and satisfaction and self-rated successful aging in older postmenopausal women. J Am Geriatr Soc 2011;59:1503-1508.

24. Kingsberg S, Krychman M, Graham S, Bernick B, Mirkin S. The Women's EMPOWER Survey: Identifying women's perceptions on vulvar and vaginal atrophy (VVA) and its treatment. J Sex Med 2017;14:413-424.

25. Kingsberg SA. Attitudinal survey of women living with low sexual desire. J Womens Health (Larchmt) 2014;23: 817-823.

26. Kingsberg SA, Wysocki S, Magnus L, Krychman ML. Vulvar and vaginal atrophy in postmenopausal women: Findings from the REVIVE (REal Women's VIews of Treatment Options for Menopausal Vaginal ChangEs) survey. J Sex Med 2013;10:1790-1799.

27. Fairchild PS, Haefner JK, Berger MB. Talk about sex: Sexual history-taking preferences among urogynecology patients and general gynecology controls. Female Pelvic Med Reconstr Surg 2016;22:297-302.

28. Feldhaus-Dahir M. Female sexual dysfunction: Barriers to treatment. Urol Nurs 2009;29:81-85; quiz 86.

29. Andrews WC. Approaches to taking a sexual history. J Womens Health Gend Based Med 2000;9 Suppl 1:S21S24.

30. Utian WH, Maamari R. Attitudes and approaches to vaginal atrophy in postmenopausal women: A focus group qualitative study. Climacteric 2014;17:29-36.

31. Maciel M, Lagana L. Older women's sexual desire problems: Biopsychosocial factors impacting them and barriers to their clinical assessment. Biomed Res Int 2014;2014: 107217.

32. Sobecki JN, Curlin FA, Rasinski KA, Lindau ST. What we don't talk about when we don't talk about sex: Results of a national survey of U.S. obstetrician/gynecologists. J Sex Med 2012;9:1285-1294.

33. Hoekstra T, Lesman-Leegte I, Couperus MF, Sanderman R, Jaarsma T. What keeps nurses from the sexual counseling of patients with heart failure? Heart Lung 2012;41:492-499.

34. Parks DM, Levine J. Helping women understand treatment options for vulvar and vaginal atrophy. Nurs Womens Health 2015;19:298-312.

35. Rosen RC, Maserejian NN, Connor MK, Krychman ML, Brown CS, Goldstein I. Characteristics of premenopausal and postmenopausal women with acquired, generalized hypoactive sexual desire disorder: The Hypoactive Sexual Desire Disorder Registry for women. Menopause 2012;19: 396-405.
36. Rossouw JE, Anderson GL, Prentice RL, et al. Risks and benefits of estrogen plus progestin in healthy postmenopausal women: Principal results From the Women's Health Initiative randomized controlled trial. JAMA 2002;288: 321-333.

37. Manson JE, Goldstein SR, Kagan R, et al. Why the product labeling for low-dose vaginal estrogen should be changed. Menopause 2014;21:911-916.

38. US Food \& Drug Administration. FDA warns against use of energy-based devices to perform vaginal 'rejuvenation' or vaginal cosmetic procedures: FDA Safety Communication. Available at: www.fda.gov/medicaldevices/safety/alertsand notices/ucm615013.htm Accessed September 26, 2018.

39. Eardley I, Reisman Y, Goldstein S, Kramer A, Dean J, Coleman E. Existing and future educational needs in graduate and postgraduate education. J Sex Med 2017;14: 475-485.

40. Shindel AW, Baazeem A, Eardley I, Coleman E. Sexual health in undergraduate medical education: Existing and future needs and platforms. J Sex Med 2016;13:1013-1026.

41. Kingsberg SA, Althof S, Simon JA, et al. Female sexual dysfunction-medical and psychological treatments, Committee 14. J Sex Med 2017;14:1463-1491.

42. Simon JA, Kingsberg SA, Shumel B, Hanes V, Garcia M, Jr., Sand M. Efficacy and safety of flibanserin in postmenopausal women with hypoactive sexual desire disorder: Results of the SNOWDROP trial. Menopause 2014;21:633-640.

43. Portman DJ, Brown L, Yuan J, Kissling R, Kingsberg SA. Flibanserin in postmenopausal women with hypoactive sexual desire disorder: Results of the PLUMERIA study. J Sex Med 2017;14:834-842.

44. Laumann EO, Waite LJ. Sexual dysfunction among older adults: Prevalence and risk factors from a nationally representative U.S. probability sample of men and women 5785 years of age. J Sex Med 2008;5:2300-2311.

45. McCabe MP, Sharlip ID, Lewis R, et al. Incidence and prevalence of sexual dysfunction in women and men: A consensus statement from the Fourth International Consultation on sexual medicine 2015. J Sex Med 2016;13: 144-152.

46. Dyspareunia: CMS clarification results in expanded treatment and coverage under Medicare Part D. PR Newswire. Available at: www.prnewswire.com/news-releases/dyspare unia-cms-clarification-results-in-expanded-treatment-andcoverage-under-medicare-part-d-300646627.html Accessed June 1, 2018.

47. ADDYI ${ }^{\circledR}$ (flibanserin) tablets $100 \mathrm{mg}$ Prescribing Information. Raleigh, NC: Sprout Pharmaceuticals, 2015.

48. Reiter S. Barriers to effective treatment of vaginal atrophy with local estrogen therapy. Int J Gen Med 2013;6:153158.

49. Nappi RE, Lachowsky M. Menopause and sexuality: Prevalence of symptoms and impact on quality of life. Maturitas 2009;63:138-141.

50. Annon JS. The PLISSIT Model: A proposed conceptual scheme for the behavioral treatment of sexual problems. J Sex Educ Ther 1976;2:1-15.

51. Wallace MA. Assessment of sexual health in older adults. Am J Nurs 2008;108:52-60; quiz 61.

52. Cole SA, Bird J. The medical interview: The three function approach, 3rd, revised ed. Amsterdam, Netherlands: Elsevier Health Sciences, 2013.

53. Rogers RG, Pauls RN, Thakar R, et al. An international Urogynecological association (IUGA)/international conti- 
nence society (ICS) joint report on the terminology for the assessment of sexual health of women with pelvic floor dysfunction. Int Urogynecol J 2018;29:647-666.

54. American College of Obstetricians and Gynecologists. Sexual health. Committee Opinion No. 706. Obstet Gynecol 2017;130:e42-e47.

55. Bober SL, Reese JB, Barbera L, et al. How to ask and what to do: A guide for clinical inquiry and intervention regarding female sexual health after cancer. Curr Opin Support Palliat Care 2016;10:44-54.

56. Hughes AK, Lewinson TD. Facilitating communication about sexual health between aging women and their health care providers. Qual Health Res 2015;25:540-550.

57. Politi MC, Clark MA, Armstrong G, McGarry KA, Sciamanna CN. Patient-provider communication about sexual health among unmarried middle-aged and older women. J Gen Intern Med 2009;24:511-516.

58. Clayton AH, Goldfischer E, Goldstein I, et al. Validity of the decreased sexual desire screener for diagnosing hypoactive sexual desire disorder. J Sex Marital Ther 2013; 39:132-143.

59. Clayton AH, Goldstein I, Kim NN, et al. The International Society for the study of women's sexual health process of care for management of hypoactive sexual desire disorder in women. Mayo Clin Proc 2018;93:467-487.

60. Goldstein I, Kim NN, Clayton AH, et al. Hypoactive sexual desire disorder: International Society for the Study of Women's Sexual Health (ISSWSH) expert consensus panel review. Mayo Clin Proc 2017;92:114-128.
61. McCabe MP, Sharlip ID, Atalla E, et al. Definitions of sexual dysfunctions in women and men: A consensus statement from the Fourth International Consultation on sexual medicine 2015. J Sex Med 2016;13:135-143.

62. Derogatis LR, Sand M, Balon R, Rosen R, Parish SJ. Toward a more evidence-based nosology and nomenclature for female sexual dysfunctions-part I. J Sex Med 2016;13: 1881-1887.

63. Parish SJ, Goldstein AT, Goldstein SW, et al. Toward a more evidence-based nosology and nomenclature for female sexual dysfunctions-part II. J Sex Med 2016;13:18881906.

64. Management of symptomatic vulvovaginal atrophy: 2013 position statement of The North American Menopause Society. Menopause 2013;20:888-902.

65. The North American Menopause Society. Sexual health. Available at: www.menopause.org/for-women/menopause flashes/sexual-health Accessed June 28, 2018.

Address correspondence to:

Sheryl A. Kingsberg, PhD

MacDonald Women's Hospital

University Hospitals Cleveland Medical Center

Mailstop 5034

11100 Euclid Avenue

Cleveland, $\mathrm{OH} 44106$

E-mail: sheryl.kingsberg@uhhospitals.org 